\title{
On Stable Perturbations of the Generalized Drazin Inverses of Closed Linear Operators in Banach Spaces
}

\author{
Qianglian Huang, ${ }^{1,2}$ Lanping $\mathrm{Zhu}^{1,2}$ \\ Xiaoru Chen, ${ }^{1}$ and Chang Zhang ${ }^{1}$ \\ ${ }^{1}$ College of Mathematics, Yangzhou University, Yangzhou 225002, China \\ 2 School of Mathematical Sciences, Monash University, Melbourne, VIC 3800, Australia \\ Correspondence should be addressed to Qianglian Huang, qlhmath@yahoo.com.cn
}

Received 15 July 2012; Accepted 28 August 2012

Academic Editor: Josef Diblík

Copyright (C) 2012 Qianglian Huang et al. This is an open access article distributed under the Creative Commons Attribution License, which permits unrestricted use, distribution, and reproduction in any medium, provided the original work is properly cited.

We investigate the stable perturbation of the generalized Drazin inverses of closed linear operators in Banach spaces and obtain some new characterizations for the generalized Drazin inverses to have prescribed range and null space. As special cases of our results, we recover the perturbation theorems of Wei and Wang, Castro and Koliha, Rakocevic and Wei, Castro and Koliha and Wei.

\section{Introduction and Preliminaries}

Let $X$ be a Banach spaces. Let $L(X), C(X)$, and $B(X)$ denote the linear space of all linear operators, the homogeneous set of all densely defined closed linear operators and the Banach space of all bounded linear operators from $X$ into itself, respectively. For any $T \in L(X)$, we denote by $D(T), N(T)$, and $R(T)$ the domain, the null space, and, respectively, the range of $T$. The identity operator will be denoted by $I$.

As an important extension of the conventional Drazin inverse, the generalized Drazin inverse in Banach algebra was introduced firstly by Koliha [1]. Later, this notion was extended to closed linear operators by Koliha and Tran [2]. Recall that an operator $S \in B(X)$ is said to be the generalized Drazin inverse of $T \in C(X)$ if $R(S) \subset D(T), R(I-T S) \subset D(T)$ and

$$
S T=T S \quad \text { on } \quad D(T), \quad S T S=S, \quad T(I-T S) \text { is quasinilpotent. }
$$


An operator $T \in C(X)$ that possesses a generalized Drazin inverse is said to be generalized Drazin invertible, and its generalized Drazin inverse is denoted by $T^{d}$. From [3-5], we know that $T$ is generalized Drazin invertible if and only if 0 is not an accumulation of $\sigma(T)$. In this case, we write $T^{\pi}=I-T T^{d}$ for the spectral idempotent of $T$.

Perturbations of the conventional Drazin inverse and the generalized Drazin inverse were studied in many papers [3-13]. For example, Castro González and Koliha [3] introduced the concept of $T$-compatible and gave the stability theorem of generalized Drazin inverse under the inverse-compatible perturbation (Theorem 1.1). Let $T \in C(X)$ be generalized Drazin invertible. An operator $\delta T \in B(X)$ is called $T$-compatible if it commutes with $T^{\pi}$ and if the operator $T^{\pi} \delta T$ is quasinilpotent and commutes with $T$, that is,

$$
T^{\pi} \delta T=\delta T T^{\pi}, \quad \sigma\left(T^{\pi} \delta T\right)=\{0\}, \quad T T^{\pi} \delta T=\delta T T T^{\pi} .
$$

Theorem 1.1 (see [3]). Let $T \in C(X)$ have the generalized Drazin inverse $T^{d} \in B(X)$ and $\delta T \in$ $B(X)$ be $T$-compatible. If $I+T^{d} \delta T: X \rightarrow X$ is invertible, then $\bar{T}=T+\delta T$ is generalized Drazin invertible and $\bar{T}^{d}=T^{d}\left(I+\delta T T^{d}\right)^{-1}=\left(I+T^{d} \delta T\right)^{-1} T^{d}$.

In this paper, we investigate the stable perturbation of the generalized Drazin inverses of closed linear operators in Banach spaces and obtain some new characterizations for the generalized Drazin inverses to have prescribed range and null space. As special cases of our results, we recover the perturbation theorems of Castro González and Koliha [3], Castro González et al. [4], Wei and Wang [6], Rakočević and Wei [7].

\section{Main Results}

First we will characterize the invertibility of $I+\delta T T^{d}$, which is inspired from [8].

Lemma 2.1. Let $T \in C(X)$ have a generalized Drazin inverse $T^{d} \in B(X)$ and $\delta T \in L(X)$ with $D(T) \subset D(\delta T)$. Then the following statements are equivalent:

(1) $I+\delta T T^{d}: X \rightarrow X$ is bijective;

(2) $I+T^{d} \delta T: D(T) \rightarrow D(T)$ is bijective;

(3) $N(\bar{T}) \cap R\left(T^{d}\right)=\{0\}$ and $X=\bar{T} R\left(T^{d}\right)+N\left(T^{d}\right)$,

where $\bar{T}=T+\delta T$ and $\dot{+}$ denotes the algebraic direct sum.

Proof. (1) $\Rightarrow(2)$. We first claim that $I+T^{d} \delta T: D(T) \rightarrow D(T)$ is injective. In fact, if $x \in D(T)$ satisfies $\left(I+T^{d} \delta T\right) x=0$, then $\delta T\left(I+T^{d} \delta T\right) x=0$, that is,

$$
\left(I+\delta T T^{d}\right) \delta T x=0
$$


Combining it with statement (1), we obtain $\delta T x=0$ and so $x=-T^{d} \delta T x=0$. Next, we will show that $I+T^{d} \delta T: D(T) \rightarrow D(T)$ is surjective, that is, for all $q \in D(T)$, we need to find a $p \in D(T)$ such that $\left(I+T^{d} \delta T\right) p=q$. Let $B=T^{d}\left(I+\delta T T^{d}\right)^{-1}$, then

$$
\begin{aligned}
\left(I+T^{d} \delta T\right) B \delta T q & =\left(I+T^{d} \delta T\right) T^{d}\left(I+\delta T T^{d}\right)^{-1} \delta T q \\
& =T^{d}\left(I+\delta T T^{d}\right)\left(I+\delta T T^{d}\right)^{-1} \delta T q=T^{d} \delta T q
\end{aligned}
$$

and $\left(I+T^{d} \delta T\right)(I-B \delta T) q=\left(I+T^{d} \delta T\right) q-T^{d} \delta T q=q$. Set $p=(I-B \delta T) q$, then $p \in D(T)$ and $\left(I+T^{d} \delta T\right) p=q$. Hence, $I+T^{d} \delta T: D(T) \rightarrow D(T)$ is bijective.

$(2) \Rightarrow(3)$. If $I+T^{d} \delta T: D(T) \rightarrow D(T)$ is bijective, let $x \in N(\bar{T}) \cap R\left(T^{d}\right)$, then there exists $y \in X$ satisfying $x=T^{d} y$ and $\bar{T} T^{d} y=\bar{T} x=0$. Hence

$$
\left(I+T^{d} \delta T\right) T^{d} y=T^{d} y+T^{d} \delta T T^{d} y=T^{d} T T^{d} y+T^{d} \delta T T^{d} y=T^{d} \bar{T} T^{d} y=0 .
$$

Thus $T^{d} y=0$, that is, $x=0$. Therefore, $N(\bar{T}) \cap R\left(T^{d}\right)=\{0\}$. Next we will show $\bar{T} R\left(T^{d}\right) \cap$ $N\left(T^{d}\right)=\{0\}$. Let $q \in \bar{T} R\left(T^{d}\right) \cap N\left(T^{d}\right)$, then there exists $p \in X$ with $q=\bar{T} T^{d} p$. Hence

$$
\begin{aligned}
0 & =T^{d} q=T^{d} \bar{T} T^{d} p=T^{d}(T+\delta T) T^{d} p \\
& =T^{d} T T^{d} p+T^{d} \delta T T^{d} p=T^{d} p+T^{d} \delta T T^{d} p \\
& =\left(I+T^{d} \delta T\right) T^{d} p .
\end{aligned}
$$

Then $T^{d} p=0$ and $q=\bar{T} T^{d} p=0$. Finally, we prove $X=\bar{T} R\left(T^{d}\right)+N\left(T^{d}\right)$. For all $x \in X$, $T^{d} x \in D(T)$. Since $I+T^{d} \delta T: D(T) \rightarrow D(T)$ is bijective, we can find $y \in D(T)$ such that $T^{d} x=\left(I+T^{d} \delta T\right) y$, that is, $T^{d} x=\left(I-T^{d} T\right) y+T^{d} \bar{T} y$. Hence

$$
T^{d}(x-\bar{T} y)=\left(I-T^{d} T\right) y \in R\left(T^{d}\right) \cap N\left(T^{d} T\right)=\{0\}
$$

Thus $y=T^{d} T y \in R\left(T^{d}\right)$ and $T^{d} x=T^{d} \bar{T} y$, which implies $x-\bar{T} y \in N\left(T^{d}\right)$. Since $x=\bar{T} y+(x-$ $\bar{T} y) \in \bar{T} R\left(T^{d}\right)+N\left(T^{d}\right)$, we obtain $X=\bar{T} R\left(T^{d}\right)+N\left(T^{d}\right)$.

(3) $\Rightarrow(1)$. If $N(\bar{T}) \cap R\left(T^{d}\right)=\{0\}$ and $X=\bar{T} R\left(T^{d}\right)+N\left(T^{d}\right)$, we first prove that $I+\delta T T^{d}$ : $X \rightarrow X$ is injective. In fact, if $x \in X$ satisfies $\left(I+\delta T T^{d}\right) x=0$, then

$$
\bar{T} T^{d} x=T T^{d} x-x \in \bar{T} R\left(T^{d}\right) \cap N\left(T^{d}\right)=\{0\}
$$

which means $x=T T^{d} x$ and $\bar{T} T^{d} x=0$. Thus $T^{d} x \in N(\bar{T}) \cap R\left(T^{d}\right)$, that is, $T^{d} x=0$. Hence $x=T T^{d} x=0$. Next, we will show that $I+\delta T T^{d}: X \rightarrow X$ is surjective. In fact, for all 
$y \in X$, since $X=\bar{T} R\left(T^{d}\right)+N\left(T^{d}\right), y$ can be expressed by $y=\bar{T} T^{d} y_{1}+y_{2}$, where $y_{1} \in X$ and $y_{2} \in N\left(T^{d}\right)$. Hence

$$
\begin{aligned}
\left(I+\delta T T^{d}\right)\left(T T^{d} y_{1}+y_{2}\right) & =\left(T T^{d} y_{1}+y_{2}\right)+(\bar{T}-T) T^{d} T T^{d} y_{1} \\
& =T T^{d} y_{1}+y_{2}+\bar{T} T^{d} y_{1}-T T^{d} y_{1} \\
& =y_{2}+\bar{T} T^{d} y_{1}=y .
\end{aligned}
$$

Therefore, $I+\delta T T^{d}: X \rightarrow X$ is bijective.

Lemma 2.2. Let $T \in C(X)$ have the generalized Drazin inverse $T^{d} \in B(X)$, and let $\delta T \in L(X)$ satisfy $D(T) \subset D(\delta T)$ and $\bar{T}=T+\delta T \in C(X)$, then $I+\delta T T^{d} \in B(X)$. Further, if $I+\delta T T^{d}: X \rightarrow X$ is bijective, then its inverse $\left(I+\delta T T^{d}\right)^{-1} \in B(X)$ and

$$
B=T^{d}\left(I+\delta T T^{d}\right)^{-1}=\left(I+T^{d} \delta T\right)^{-1} T^{d}: X \longrightarrow X
$$

is a well-defined bounded linear operator with $R(B)=R\left(T^{d}\right)$ and $N(B)=N\left(T^{d}\right)$.

Proof. Since $T^{d}$ is bounded and $\bar{T}$ is closed, by the Closed Operator Theorem, we know that $\bar{T} T^{d}$ is bounded and so is $I+\delta T T^{d}=I-T T^{d}+\bar{T} T^{d}$. Further, if $I+\delta T T^{d}: X \rightarrow X$ is bijective, it follows from the Inverse Operator Theorem that $\left(I+\delta T T^{d}\right)^{-1} \in B(X)$. By Lemma 2.1 and $\left(I+T^{d} \delta T\right) T^{d}=T^{d}\left(I+\delta T T^{d}\right)$, we can see that

$$
B=T^{d}\left(I+\delta T T^{d}\right)^{-1}=\left(I+T^{d} \delta T\right)^{-1} T^{d}: X \longrightarrow R\left(T^{d}\right)
$$

is a well-defined bounded linear operator. Easily, we can obtain $R(B)=R\left(T^{d}\right)$ and $N(B)=$ $N\left(T^{d}\right)$.

The following theorem points out that the generalized Drazin inverse with the prescribed range and null space must possess the expression $T^{d}\left(I+\delta T T^{d}\right)^{-1}$.

Theorem 2.3. Let $T \in C(X)$ have the generalized Drazin inverse $T^{d} \in B(X)$, and let $\delta T \in L(X)$ satisfy $D(T) \subset D(\delta T)$ and $\bar{T}=T+\delta T \in C(X)$. Then the following statements are equivalent:

(1) $\bar{T}$ is generalized Drazin invertible with $\bar{T}^{\pi}=T^{\pi}$;

(2) $\bar{T}$ is generalized Drazin invertible with $N\left(\bar{T}^{d}\right)=N\left(T^{d}\right)$ and $R\left(\bar{T}^{d}\right)=R\left(T^{d}\right)$;

(3) $I+\delta T T^{d}: X \rightarrow X$ is bijective and

$$
B=T^{d}\left(I+\delta T T^{d}\right)^{-1}=\left(I+T^{d} \delta T\right)^{-1} T^{d}
$$

is the generalized Drazin inverse of $\bar{T}$;

(4) $\bar{T}$ is generalized Drazin invertible with $T^{d}-\bar{T}^{d}=T^{d} \delta T \bar{T}^{d}=\bar{T}^{d} \delta T T^{d}$;

(5) $\bar{T}$ is generalized Drazin invertible with $T^{d}-\bar{T}^{d}=\bar{T}^{d} \delta T T^{d}$. 
Proof. It is easy to see (1) $\Rightarrow(2)$ and $(4) \Rightarrow(5)$.

$(2) \Rightarrow(3)$. Suppose that $\bar{T}$ is generalized Drazin invertible with $N\left(\bar{T}^{d}\right)=N\left(T^{d}\right)$ and $R\left(\bar{T}^{d}\right)=R\left(T^{d}\right)$. We first show that $I+\delta T T^{d}: X \rightarrow X$ is bijective. From Lemma 2.1, it suffices to show $N(\bar{T}) \cap R\left(T^{d}\right)=\{0\}$ and $X=\bar{T} R\left(T^{d}\right)+N\left(T^{d}\right)$. Since $\bar{T}^{d}$ is the generalized Drazin inverse of $\bar{T}$, we can obtain $N(\bar{T}) \cap R\left(\bar{T}^{d}\right)=\{0\}$ and $X=R\left(\overline{T T}^{d}\right) \oplus N\left(\overline{T T}^{d}\right)=\bar{T} R\left(\bar{T}^{d}\right) \oplus N\left(\bar{T}^{d}\right)$. Hence

$$
N(\bar{T}) \cap R\left(T^{d}\right)=\{0\}, \quad X=\bar{T} R\left(T^{d}\right) \oplus N\left(T^{d}\right)
$$

Next, by $N\left(\bar{T}^{d}\right)=N\left(T^{d}\right)$ and $R\left(\bar{T}^{d}\right)=R\left(T^{d}\right)$, we can obtain $\bar{T}^{d}\left(I-T T^{d}\right)=0$ and $\left(\bar{T}^{d} \bar{T}-I\right) T^{d}=$ 0 , that is,

$$
\bar{T}^{d}=\bar{T}^{d} T T^{d}, \quad \bar{T}^{d} \bar{T}^{d}=T^{d}
$$

Then $\bar{T}^{d}+\bar{T}^{d} \bar{T}^{d}-\bar{T}^{d} T T^{d}=T^{d}$, that is,

$$
\bar{T}^{d}\left(I+\delta T T^{d}\right)=\bar{T}^{d}\left[I+(\bar{T}-T) T^{d}\right]=T^{d} .
$$

Therefore, $\bar{T}^{d}=T^{d}\left(I+\delta T T^{d}\right)^{-1}$. By Lemma 2.2, we have $\bar{T}^{d}=\left(I+T^{d} \delta T\right)^{-1} T^{d}$.

$(3) \Rightarrow(4)$. Since the generalized Drazin inverse is unique, we get

$$
\bar{T}^{d}=T^{d}\left(I+\delta T T^{d}\right)^{-1}=\left(I+T^{d} \delta T\right)^{-1} T^{d} .
$$

Then $\bar{T}^{d}\left(I+\delta T T^{d}\right)=T^{d}$ and $\left(I+T^{d} \delta T\right) \bar{T}^{d}=T^{d}$. Hence

$$
T^{d}-\bar{T}^{d}=\bar{T}^{d} \delta T T^{d}=T^{d} \delta T \bar{T}^{d} .
$$

(5) $\Rightarrow(1)$. We only need to prove $\overline{T T}^{d}=T T^{d}$. In fact, by $T^{d}-\bar{T}^{d}=\bar{T}^{d} \delta T T^{d}$, we obtain $T^{d}=\bar{T}^{d}\left(I+\delta T T^{d}\right)$ and $\bar{T}^{d}=\left(I-\bar{T}^{d} \delta T\right) T^{d}$. Hence

$$
\begin{gathered}
\bar{T}^{d} \bar{T}^{d}=\bar{T}^{d} \overline{T T}^{d}\left(I+\delta T T^{d}\right)=\bar{T}^{d}\left(I+\delta T T^{d}\right)=T^{d}, \\
\bar{T}^{d} T T^{d}=\left(I-\bar{T}^{d} \delta T\right) T^{d} T T^{d}=\left(I-\bar{T}^{d} \delta T\right) T^{d}=\bar{T}^{d} .
\end{gathered}
$$

Thus

$$
\overline{T T}^{d}=\overline{T T}^{d} T T^{d}=\overline{T T}^{d} T^{d} T=\bar{T}^{d} \bar{T}^{d} T=T^{d} T=T T^{d} \quad \text { on } \quad D(T) .
$$

Since $D(T)$ is dense in $X, \overline{T T}^{d}$ and $T T^{d}$ are bounded, we can get $\overline{T T}^{d}=T T^{d}$ and so $\bar{T}^{\pi}=$ $I-\overline{T T}^{d}=I-T T^{d}=T^{\pi}$. 
Remark 2.4. To guarantee $\bar{T} \in C(X)$, a general condition of $T$ boundedness is often assumed, see [14].

Theorem 2.5. Let $T \in C(X)$ have the generalized Drazin inverse $T^{d} \in B(X)$ and $\delta T \in L(X)$ satisfy $D(T) \subset D(\delta T)$ and $\bar{T}=T+\delta T \in C(X)$. Assume that $I+\delta T T^{d}: X \rightarrow X$ is bijective, then the following statements are equivalent:

(1)

$$
B=T^{d}\left(I+\delta T T^{d}\right)^{-1}=\left(I+T^{d} \delta T\right)^{-1} T^{d}
$$

is the generalized Drazin inverse of $\bar{T}$;

(2) $\bar{T} T^{d}=T T^{d} \bar{T} T^{d}, T^{d} \bar{T}=T^{d} \bar{T} T^{d} T$ and $\lim _{n \rightarrow+\infty}\left\|\bar{T}^{n}\left(I-T^{d} T\right)\right\|^{1 / n}=0$;

(3) $\bar{T} T^{d} T=T T^{d} \bar{T}$ and $\lim _{n \rightarrow+\infty}\left\|\bar{T}^{n}\left(I-T^{d} T\right)\right\|^{1 / n}=0$;

(4) $\bar{T} T^{d} T=T T^{d} \bar{T}$ and $\bar{T} T^{\pi}$ is quasinilpotent;

(5) $\bar{T} T^{d}=T T^{d} \bar{T} T^{d}, T^{d} \bar{T}=T^{d} \bar{T} T^{d} T$ and $\bar{T} T^{\pi}$ is quasinilpotent.

In this case, $\bar{T}^{\pi}=T^{\pi}$ and

$$
\left\|\bar{T}^{d}-T^{d}\right\| \leq\left\|T^{d}\right\| \cdot\left\|\delta T T^{d}\right\| \cdot\left\|\left(I+\delta T T^{d}\right)^{-1}\right\| .
$$

Proof. (1) $\Rightarrow$ (2). If $B$ is the generalized Drazin inverse of $\bar{T}$, then by Theorem 2.3, we get $\bar{T}^{\pi}=T^{\pi}$ and so $\bar{T} B=T T^{d}$ and $B \bar{T}=T^{d} T$. Hence $\bar{T} T^{d}=T T^{d}\left[I+(\bar{T}-T) T^{d}\right]=T T^{d} \bar{T} T^{d}$ and $T^{d} \bar{T}=\left[I+T^{d}(\bar{T}-T)\right] T^{d} T=T^{d} \bar{T} T^{d} T$. Also, for all $x \in D(\bar{T})$,

$$
\begin{aligned}
{[\bar{T}(I-\bar{T} B)]^{2} x } & =[\bar{T}(I-\bar{T} B) \bar{T}(I-\bar{T} B)] x \\
& =\bar{T}(I-\bar{T} B) \bar{T}(I-B \bar{T}) x \\
& =\overline{T T}(I-B \bar{T})(I-B \bar{T}) x \\
& =\bar{T}^{2}(I-B \bar{T}) x \\
& =\bar{T}^{2}\left(I-T^{d} T\right) x
\end{aligned}
$$

Thus we obtain $\bar{T}(I-B \bar{T}) x=\bar{T} x-\bar{T} B \bar{T} x=(I-\bar{T} B) \bar{T} x \in D(\bar{T})$ and

$$
\begin{aligned}
{[\bar{T}(I-\bar{T} B)]^{3} x } & =[\bar{T}(I-\bar{T} B)]^{2}[\bar{T}(I-\bar{T} B)] x \\
& =[\bar{T}(I-\bar{T} B)]^{2}[\bar{T}(I-B \bar{T})] x
\end{aligned}
$$


Abstract and Applied Analysis

$$
\begin{aligned}
& =\bar{T}^{2}(I-B \bar{T}) \bar{T}(I-B \bar{T}) x \\
& =\bar{T}^{2}(I-\bar{T} B) \bar{T}(I-B \bar{T}) x \\
& =\bar{T}^{3}(I-B \bar{T})(I-B \bar{T}) x \\
& =\bar{T}^{3}(I-B \bar{T}) x \\
& =\bar{T}^{3}\left(I-T^{d} T\right) x .
\end{aligned}
$$

Hence for all $n \in N$,

$$
[\bar{T}(I-\bar{T} B)]^{n}=\bar{T}^{n}\left(I-T^{d} T\right) \quad \text { on } \quad D(T)
$$

Therefore $\lim _{n \rightarrow+\infty}\left\|\bar{T}^{n}\left(I-T^{d} T\right)\right\|^{1 / n}=\lim _{n \rightarrow+\infty}\left\|[\bar{T}(I-\bar{T} B)]^{n}\right\|^{1 / n}=0$.

$(2) \Rightarrow(1)$. It follows from Lemma 2.2 that $B=T^{d}\left(I+\delta T T^{d}\right)^{-1}=\left(I+T^{d} \delta T\right)^{-1} T^{d}$ is a well-defined bounded linear operator with $R(B)=R\left(T^{d}\right) \subset D(T)=D(\bar{T})$. By $\bar{T} T^{d}=T T^{d} \bar{T} T^{d}$ and $T^{d} \bar{T}=T^{d} \bar{T} T^{d} T$, we can verify

$$
\begin{aligned}
\bar{T} B & =\bar{T} T^{d}\left(I+\delta T T^{d}\right)^{-1} \\
& =T T^{d} \bar{T} T^{d}\left(I+\delta T T^{d}\right)^{-1} \\
& =T T^{d}(T+\delta T) T^{d}\left(I+\delta T T^{d}\right)^{-1} \\
& =\left(T T^{d}+T T^{d} \delta T T^{d}\right)\left(I+\delta T T^{d}\right)^{-1} \\
& =T T^{d}\left(I+\delta T T^{d}\right)\left(I+\delta T T^{d}\right)^{-1}=T T^{d}
\end{aligned}
$$

and by Lemma 2.1,

$$
\begin{aligned}
B \bar{T} & =\left(I+T^{d} \delta T\right)^{-1} T^{d} \bar{T} \\
& =\left(I+T^{d} \delta T\right)^{-1} T^{d} \bar{T} T^{d} T \\
& =\left(I+T^{d} \delta T\right)^{-1} T^{d}(T+\delta T) T^{d} T \\
& =\left(I+T^{d} \delta T\right)^{-1}\left(T^{d} T+T^{d} \delta T\right) T^{d} T \\
& =\left(I+T^{d} \delta T\right)^{-1}\left(I+T^{d} \delta T\right) T^{d} T=T^{d} T .
\end{aligned}
$$


Thus $\bar{T} B=B \bar{T}$ on $D(\bar{T})$. Obviously, $R(I-\bar{T} B)=R\left(I-T T^{d}\right) \subset D(T)=D(\bar{T})$. Also, we can see

$$
\begin{aligned}
B \bar{T} B & =T^{d}\left(I+\delta T T^{d}\right)^{-1} \bar{T} T^{d}\left(I+\delta T T^{d}\right)^{-1} \\
& =T^{d}\left(I+\delta T T^{d}\right)^{-1}(T+\delta T) T^{d}\left(I+\delta T T^{d}\right)^{-1} \\
& =T^{d}\left(I+\delta T T^{d}\right)^{-1}\left(T T^{d}+\delta T T^{d} T T^{d}\right)\left(I+\delta T T^{d}\right)^{-1} \\
& =T^{d}\left(I+\delta T T^{d}\right)^{-1}\left(I+\delta T T^{d}\right) T T^{d}\left(I+\delta T T^{d}\right)^{-1} \\
& =T^{d}\left(I+\delta T T^{d}\right)^{-1}=B .
\end{aligned}
$$

To the aim, we need show $\bar{T}(I-\bar{T} B)$ is quasinilpotent. In fact, as in $(1) \Rightarrow(2)$, we can obtain (2.22) and so

$$
\lim _{n \rightarrow+\infty}\left\|[\bar{T}(I-\bar{T} B)]^{n}\right\|^{1 / n}=\lim _{n \rightarrow+\infty}\left\|\bar{T}^{n}\left(I-T^{d} T\right)\right\|^{1 / n}=0 .
$$

Therefore, $B$ is the generalized Drazin inverse of $\bar{T}$.

(2) $\Leftrightarrow$ (3). If $\bar{T} T^{d}=T T^{d} \bar{T} T^{d}$ and $T^{d} \bar{T}=T^{d} \bar{T} T^{d} T$, then

$$
\bar{T} T^{d} T=T T^{d} \bar{T} T^{d} T=T T^{d} \bar{T} .
$$

Conversely, if $\bar{T} T^{d} T=T T^{d} \bar{T}$, then

$$
\bar{T} T^{d}=\bar{T} T^{d} T T^{d}=T T^{d} \bar{T} T^{d}, \quad T^{d} \bar{T}=T^{d} T T^{d} \bar{T}=T^{d} \bar{T} T^{d} T .
$$

(3) $\Leftrightarrow$ (4). It follows from $\bar{T} T^{d} T=T T^{d} \bar{T}$ that

$$
\begin{aligned}
\left(\bar{T} T^{\pi}\right)^{2} & =\bar{T} T^{\pi} \bar{T} T^{\pi}=\bar{T}\left(I-T T^{d}\right) \bar{T} T^{\pi} \\
& =\bar{T}\left(\bar{T}-T T^{d} \bar{T}\right) T^{\pi}=\bar{T}\left(\bar{T}-\bar{T} T^{d} T\right) T^{\pi} \\
& =\bar{T}^{2}\left(I-T^{d} T\right) T^{\pi}=\bar{T}^{2}\left(I-T^{d} T\right) \quad \text { on } D(T)
\end{aligned}
$$

and then for all $n \in N$,

$$
\left(\bar{T} T^{\pi}\right)^{n}=\bar{T}^{n}\left(I-T^{d} T\right) \quad \text { on } \quad D(T)
$$

Therefore, $\bar{T} T^{\pi}$ is quasinilpotent if and only if $\lim _{n \rightarrow+\infty}\left\|\bar{T}^{n}\left(I-T^{d} T\right)\right\|^{1 / n}=0$. 
$(4) \Leftrightarrow(5)$. It is similar to (2) $\Leftrightarrow(3)$. In this case,

$$
\begin{aligned}
\left\|B-T^{d}\right\| & =\left\|T^{d}\left(I+\delta T T^{d}\right)^{-1}-T^{d}\right\| \\
& \leq\left\|T^{d} \delta T T^{d}\left(I+\delta T T^{d}\right)^{-1}\right\| \\
& \leq\left\|T^{d}\right\| \cdot\left\|\delta T T^{d}\right\| \cdot\left\|\left(I+\delta T T^{d}\right)^{-1}\right\| .
\end{aligned}
$$

Remark 2.6. Theorems 2.3 and 2.5 generalize the main results in $[3-8,10,12,13]$.

As applications, we recover some known results in $[3,6,7]$.

Corollary 2.7 (see [3]). Let $T \in C(X)$ have the generalized Drazin inverse $T^{d} \in B(X)$ and $\delta T \in$ $B(X)$ be $T$-compatible. If $I+T^{d} \delta T: X \rightarrow X$ is invertible, then $\bar{T}=T+\delta T$ is generalized Drazin invertible and $\bar{T}^{d}=T^{d}\left(I+\delta T T^{d}\right)^{-1}=\left(I+T^{d} \delta T\right)^{-1} T^{d}$.

Proof. It is easy to see $\bar{T} \in C(X)$ and $\bar{T} T^{d} T=T T^{d} \bar{T}$. Since

$$
\bar{T} T^{\pi}=T T^{\pi}+\delta T T^{\pi}
$$

is the sum of two commuting quasinilpotent operators, we know that $\bar{T} T^{\pi}$ is quasinilpotent. Hence by Theorem 2.5, we can get what we desired.

Corollary 2.8. Let $T \in C(X)$ have the generalized Drazin inverse $T^{d} \in B(X)$ and $\delta T \in L(X)$ satisfy $D(T) \subset D(\delta T)$ and $\bar{T}=T+\delta T \in C(X)$. Assume that $I+\delta T T^{d}: X \rightarrow X$ is bijective and

$$
\delta T=T T^{d} \delta T=\delta T T^{d} T
$$

then $B=T^{d}\left(I+\delta T T^{d}\right)^{-1}=\left(I+T^{d} \delta T\right)^{-1} T^{d}$ is the generalized Drazin inverse of $\bar{T}=T+\delta T$.

Proof. If $\delta T=T T^{d} \delta T=\delta T T^{d} T$, then $\bar{T} T^{d} T=T T^{d} \bar{T}$ and $\bar{T} T^{\pi}=T T^{\pi}$ is quasinilpotent. From Theorem 2.5, $B$ is the generalized Drazin inverse of $\bar{T}$.

Remark 2.9. The condition $\delta T=T T^{D} \delta T=\delta T T^{D} T$ is called the condition $(W)$ in $[6,7]$, which appear as a sufficient condition to ensure that $T^{D}\left(I+\delta T T^{D}\right)^{-1}$ is the Drazin inverse of $\bar{T}$.

The following theorem shows that the above condition $(W)$ is exactly the stable characterization of group inverse. Recall that an operator $S \in B(X)$ is said to be the group inverse of $T \in C(X)$ if $S$ satisfies $R(S) \subset D(T)$ and

$$
T S T=T ; \quad S T S=S, \quad S T=T S \quad \text { on } \quad D(T) .
$$

The group inverse of $T$ is unique if it exists, which is always denoted by $T^{\#}$. 
Theorem 2.10. Let $T \in C(X)$ have the group inverse $T^{\#} \in B(X)$ and let $\delta T \in L(X)$ satisfy $D(T) \subset$ $D(\delta T)$ and $\bar{T}=T+\delta T \in C(X)$. Assume that $I+\delta T T^{\#}: X \rightarrow X$ is bijective, then

$$
B=T^{\#}\left(I+\delta T T^{\#}\right)^{-1}=\left(I+T^{\#} \delta T\right)^{-1} T^{\#}
$$

is the group inverse of $\bar{T}$ if and only if

$$
\delta T=T T^{\#} \delta T=\delta T T^{\#} T .
$$

In this case, $\bar{T}^{\pi}=T^{\pi}, R(\bar{T})=R(T)$ and $N(\bar{T})=N(T)$.

Proof. If $\delta T=T T^{\#} \delta T=\delta T T^{\#} T$, then $\bar{T}=\bar{T} T^{\#} T=T T^{\#} \bar{T}$ and

$$
\bar{T} T^{\pi}=\bar{T}\left(I-T T^{\#}\right)=\bar{T}\left(I-T^{\#} T\right)=0 \quad \text { on } \quad D(T) .
$$

Hence $\bar{T} T^{\pi}=0$. And by (4) in Theorem 2.5, we know that $B$ is the generalized Drazin inverse of $\bar{T}$ with $\bar{T} B=T T^{\#}$ and $B \bar{T}=T^{\#} T$. Hence $\bar{T} B \bar{T}=T T^{\#} \bar{T}=\bar{T}$. Therefore, $B$ is also the group inverse of $\bar{T}$. Conversely, if $B$ is the group inverse of $\bar{T}$, then

$$
\begin{gathered}
R(\bar{T})=R(\bar{T} B)=\overline{R(B \bar{T})}=\overline{R\left(T^{\#} T\right)}=R\left(T T^{\#}\right)=R(T), \\
N(\bar{T})=N(B \bar{T})=N(\bar{T} B) \cap D(\bar{T})=N\left(T T^{\#}\right) \cap D(T)=N\left(T^{\#} T\right)=R\left(I-T^{\#} T\right) .
\end{gathered}
$$

Hence

$$
\begin{aligned}
& \bar{T}-T T^{\#} \bar{T}=\left(I-T T^{\#}\right) \bar{T}=0, \\
& \bar{T}-\bar{T} T^{\#} T=\bar{T}\left(I-T^{\#} T\right)=0 .
\end{aligned}
$$

Thus $\bar{T}=T T^{\#} \bar{T}=\bar{T} T^{\#} T$, that is, $\delta T=T T^{\#} \delta T=\delta T T^{\#} T$.

The following corollary extends the Proposition 4.2 in [3] to the case of closed linear operators. Recall that $T \in C(X)$ is an $E P$ (Equal Projections) operator if $T$ has the MoorePenrose inverse $T^{\dagger} \in B(X)$ satisfying $T^{\dagger} T=T T^{\dagger}$ on $D(T)$.

Corollary 2.11. Let $X$ be a Hilbert space and $T \in C(X)$ be an EP operator. Let $\delta T \in L(X)$ satisfy $D(T) \subset D(\delta T)$ and $\bar{T}=T+\delta T \in C(X)$. Assume that $I+\delta T T^{\dagger}: X \rightarrow X$ is bijective, then $\bar{T}$ is also an EP operator and

$$
B=T^{\dagger}\left(I+\delta T T^{\dagger}\right)^{-1}=\left(I+T^{\dagger} \delta T\right)^{-1} T^{\dagger}
$$


is its Moore-Penrose inverse if and only if

$$
\delta T=T T^{\dagger} \delta T=\delta T T^{\dagger} T .
$$

In this case, $B \bar{T}=\bar{T} B$ on $D(\bar{T}), \bar{T}^{\pi}=T^{\pi}, R(\bar{T})=R(T)$ and $N(\bar{T})=N(T)$.

Proof. If $\bar{T}$ is an EP operator and $B$ is its Moore-Penrose inverse, then $B$ is also its group inverse. By Theorem 2.10, we get $\delta T=T T^{\dagger} \delta T=\delta T T^{\dagger} T$. Conversely, if $\delta T=T T^{\dagger} \delta T=\delta T T^{\dagger} T$, then by Theorem 2.10 again, $B$ is the group inverse of $\bar{T}, \bar{T} B=T T^{\dagger}$ and $B \bar{T}=T^{\dagger} T$. Therefore, $B$ is the Moore-Penrose inverse of $\bar{T}$.

To illustrate our results, we give an example of a closed EP operator.

Example 2.12 (see [15]). Let

$$
L^{2}[0,1]=\left\{f, f \text { measurable complex }- \text { valued on }[0,1], \int_{[0,1]}|f(x)|^{2} d x<\infty\right\}
$$

be the Hilbert space with the inner product:

$$
\langle f, g\rangle=\int_{[0,1]} \overline{f(x)} g(x) d x, \quad f, g \in L^{2}[0,1]
$$

Set $t:[0,1] \rightarrow C$ by

$$
t(x)=\left\{\begin{array}{lr}
1 & x=0 \\
\frac{1}{\sqrt{x}} & 0<x \leq 1
\end{array}\right.
$$

and define the maximal operator of multiplication $T$ by $t$ on $L^{2}[0,1]$, that is,

$$
T f=t f, \quad \text { for } f \in D(T)=\left\{f \in L^{2}[0,1], t f \in L^{2}[0,1]\right\} \text {, }
$$

then $T$ is a densely defined closed linear operator [16]. Since $|t(x)| \geq 1$, for all $x \in[0,1]$, $R(T)=L^{2}[0,1]$ and $T$ has a bounded inverse $T^{-1}: L^{2}[0,1] \rightarrow L^{2}[0,1]$ defined by

$$
T^{-1} g=t_{1} g, \quad \forall g \in L^{2}[0,1]
$$

where

$$
t_{1}(x)= \begin{cases}1 & x=0 \\ \sqrt{x} & 0<x \leq 1\end{cases}
$$

Therefore, $T$ is a closed operator on $L^{2}[0,1]$ with a bounded inverse $T^{-1}$, which is both the Moore-Penrose inverse and the group inverse of $T$. 


\section{Acknowledgments}

The authors would like to express their deep gratitude to the referee for his/her constructive comments and suggestions. This research is supported by the National Natural Science Foundation of China (11201410 and 11271316), the Natural Science Foundation of Jiangsu province (BK2010309 and BK2012260), the Jiangsu Government Scholarship for Overseas Studies, the Natural Science Foundation of Jiangsu Education Committee (10KJB110012), the Scholarship for Overseas Studies, and the Natural Science Foundation of Yangzhou University.

\section{References}

[1] J. J. Koliha, “A generalized Drazin inverse," Glasgow Mathematical Journal, vol. 38, no. 3, pp. 367-381, 1996.

[2] J. J. Koliha and T. D. Tran, "The Drazin inverse for closed linear operators and the asymptotic convergence of $C_{0}$-semigroups," Journal of Operator Theory, vol. 46, no. 2, pp. 323-336, 2001.

[3] N. Castro González and J. J. Koliha, "Perturbation of the Drazin inverse for closed linear operators," Integral Equations and Operator Theory, vol. 36, no. 1, pp. 92-106, 2000.

[4] N. Castro González, J. J. Koliha, and Y. Wei, "Error bounds for perturbation of the Drazin inverse of closed operators with equal spectral projections," Applicable Analysis, vol. 81, no. 4, pp. 915-928, 2002.

[5] N. Castro González, J. J. Koliha, and V. Rakočević, "Continuity and general perturbation of the Drazin inverse for closed linear operators," Abstract and Applied Analysis, vol. 7, no. 6, pp. 335-347, 2002.

[6] Y. Wei and G. Wang, "The perturbation theory for the Drazin inverse and its applications," Linear Algebra and Its Applications, vol. 258, pp. 179-186, 1997.

[7] V. Rakočević and Y. Wei, "The perturbation theory for the Drazin inverse and its applications. II," Journal of the Australian Mathematical Society, vol. 70, no. 2, pp. 189-197, 2001.

[8] Q. Huang, L. Zhu, and Y. Jiang, "On stable perturbations for outer inverses of linear operators in Banach spaces," Linear Algebra and Its Applications, vol. 437, no. 7, pp. 1942-1954, 2012.

[9] J. J. Koliha and V. Rakočević, "Continuity of the Drazin inverse. II," Studia Mathematica, vol. 131, no. 2, pp. 167-177, 1998.

[10] Y. Wei and H. Wu, "The perturbation of the Drazin inverse and oblique projection," Applied Mathematics Letters, vol. 13, no. 3, pp. 77-83, 2000.

[11] N. Castro-González and J. Y. Vélez-Cerrada, "On the perturbation of the group generalized inverse for a class of bounded operators in Banach spaces," Journal of Mathematical Analysis and Applications, vol. 341, no. 2, pp. 1213-1223, 2008.

[12] Q. Xu, C. Song, and Y. Wei, "The stable perturbation of the Drazin inverse of the square matrices," SIAM Journal on Matrix Analysis and Applications, vol. 31, no. 3, pp. 1507-1520, 2009.

[13] Q. Huang, L. Zhu, W. Geng, and J. Yu, "Perturbation and expression for inner inverses in Banach spaces and its applications," Linear Algebra and Its Applications, vol. 436, no. 9, pp. 3721-3735, 2012.

[14] T. Kato, Perturbation Theory for Linear Operators, Springer, Berlin, Germany, 1984.

[15] Q. Huang, L. Zhu, and J. Yu, "Some new perturbation results for generalized inverses of closed linear operators in Banach spaces," Banach Journal of Mathematical Analysis, vol. 6, no. 2, pp. 58-68, 2012.

[16] J. Weidmann, Linear Operators in Hilbert Spaces, vol. 68 of Graduate Texts in Mathematics, Springer, New York, NY, USA, 1980. 


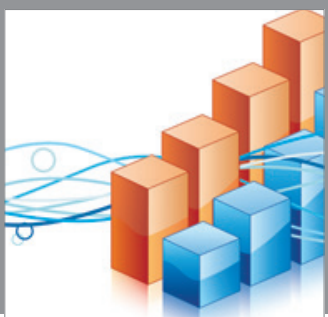

Advances in

Operations Research

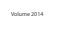

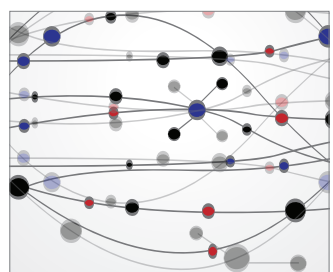

\section{The Scientific} World Journal
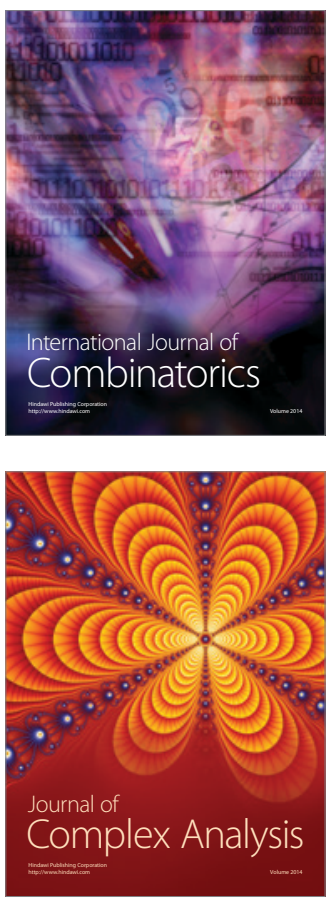

International Journal of

Mathematics and

Mathematical

Sciences
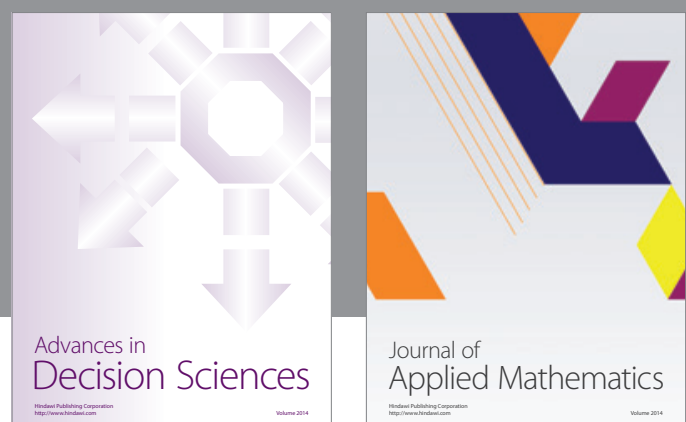

Journal of

Applied Mathematics
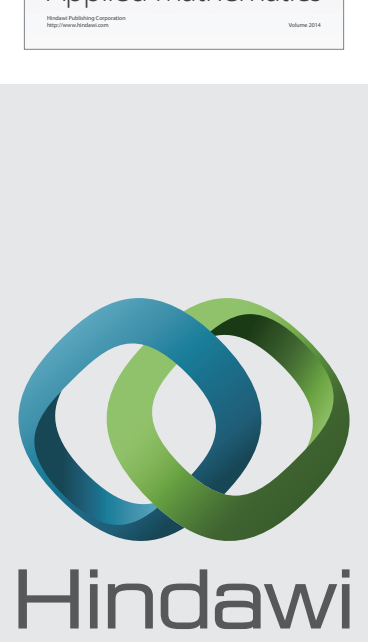

Submit your manuscripts at http://www.hindawi.com
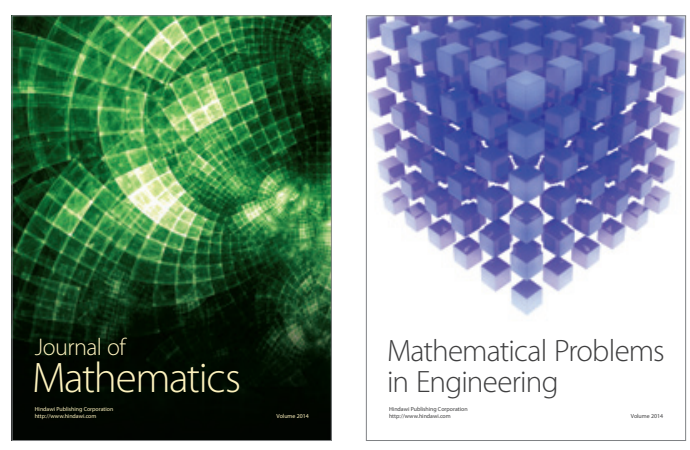

Mathematical Problems in Engineering
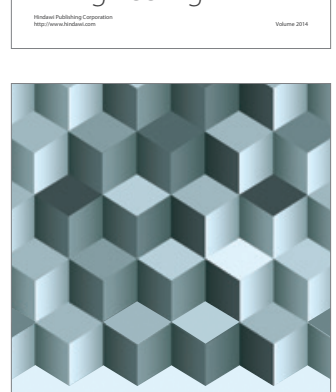

Journal of

Function Spaces
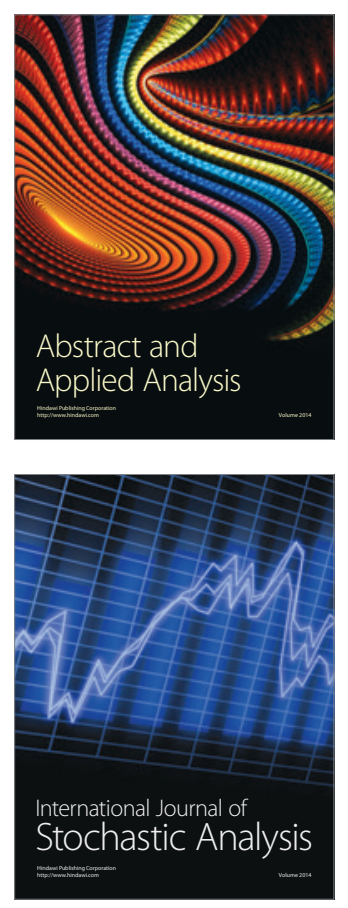

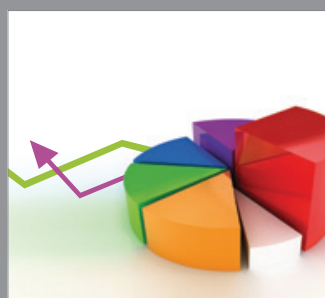

ournal of

Probability and Statistics

Promensencen
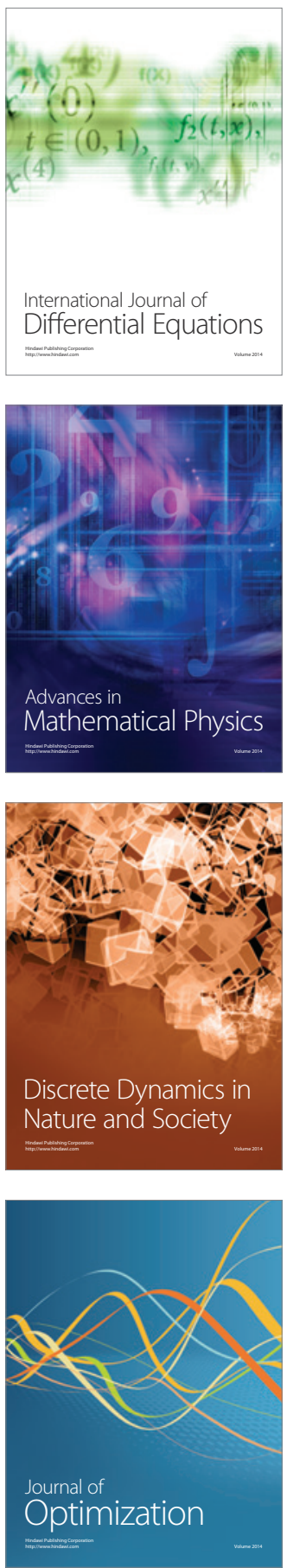